\title{
Modulation of Perineuronal Nets and Parvalbumin with Developmental Song Learning
}

\author{
Timothy S. Balmer, ${ }^{\star}$ Vanessa M. Carels, ${ }^{\star}$ Jillian L. Frisch, and Teresa A. Nick \\ Department of Neuroscience and Center for Neurobehavioral Development, University of Minnesota Academic Health Center, Minneapolis, Minnesota 55455
}

\begin{abstract}
Neural circuits and behavior are shaped during developmental phases of maximal plasticity known as sensitive or critical periods. Neural correlates of sensory critical periods have been identified, but their roles remain unclear. Factors that define critical periods in sensorimotor circuits and behavior are not known. Birdsong learning in the zebra finch occurs during a sensitive period similar to that for human speech. We now show that perineuronal nets, which correlate with sensory critical periods, surround parvalbumin-positive neurons in brain areas that are dedicated to singing. The percentage of both total and parvalbumin-positive neurons with perineuronal nets increased with development. In HVC (this acronym is the proper name), a song area important for sensorimotor integration, the percentage of parvalbumin neurons with perineuronal nets correlated with song maturity. Shifting the vocal critical period with tutor song deprivation decreased the percentage of neurons that were parvalbumin positive and the relative staining intensity of both parvalbumin and a component of perineuronal nets. Developmental song learning shares key characteristics with sensory critical periods, suggesting shared underlying mechanisms.
\end{abstract}

\section{Introduction}

During sensitive periods, enhanced neural plasticity enables environmental factors to shape developing circuits and behavior (Wiesel and Hubel, 1963; Knudsen, 2004; Hensch, 2005). Four cellular features characterize the primary visual cortex during the critical period for ocular dominance plasticity (Hensch, 2005): prolonged bursting, expression of the calcium-binding protein parvalbumin $(\mathrm{PV})$ that is characteristic of fast-spiking neurons, typically interneurons (Celio, 1986; Wild et al., 2005), extracellular matrix perineuronal nets (PNNs) (Hockfield and McKay, 1983; Sur et al., 1988), and changes in excitatory-inhibitory balance. All of these features relate to inhibitory interneurons, because facilitation of GABA neurotransmission decreases the incidence of prolonged bursts (Fagiolini and Hensch, 2000), and $\mathrm{PV}$ and PNNs preferentially associate with GABA-expressing interneurons (Celio, 1986; Braun et al., 1988). Prolonged discharge

Received June 23, 2009; revised Aug. 10, 2009; accepted Sept. 1, 2009.

This work was supported by The John Merck Scholars Program, National Institute on Deafness and Other Communication Disorders Grants R01-DC007384 and K02-DC008521, the National Institute of Neural Disease and Stroke Grant R01-NS050436, the Minnesota Medical Foundation, The Grant-in-Aid of Research, Artistry, and Scholarship from the University of Minnesota Graduate School (T.A.N.), and the University of Minnesota Undergraduate Research Opportunities Program (V.M.C., J.L.F.). We thank M. Coleman for expert assistance in locating the song nucleus Uva. We thank T. Hensch, P. Letourneau, S. McCloon, Y. Nakagawa, J. Sedgewick, and M. Wessendorf for helpful advice and T. Hensch, F. Kubke, L. Lanier, J. Sedgewick, M. Wessendorf, and Y. Yazaki-Sugiyama for reviewing preliminary drafts of this manuscript. T.S.B. and T.A.N. discovered perineuronal nets in the song system and their developmental regulation. T.S.B. and V.M.C. developed all key histological methods. T.A.N. wrote all custom analytical software T.S.B., V.M.C., J.L.F., and T.A.N. collected, processed, and analyzed histological and behavioral data for the developmental and behavioral studies. V.M.C. and J.L.F. executed the isolate study and optimized the fixation procedure T.S.B. and V.M.C. contributed equally to the study. T.A.N. wrote the manuscript, and all authors discussed the results and commented on the manuscript.

*T.S.B. and V.M.C. contributed equally to this work.

Correspondence should be addressed to Teresa A. Nick, 6-145 Jackson Hall, 321 Church Street SE, Minneapolis, MN 55455. E-mail: nickx002@umn.edu.

T. S. Balmer's present address: Department of Biology, Georgia State University, 402 Kell Hall, Atlanta, GA 30302-4010.

DOI:10.1523/JNEUROSCI.2974-09.2009

Copyright $\odot 2009$ Society for Neuroscience $\quad$ 0270-6474/09/2912878-08\$15.00/0 is thought to reflect an immature state of neural circuitry, whereas increased inhibitory interneuron activity and the appearance of PV marks the onset of experience-dependent plasticity. The appearance of PNNs then heralds the end of the critical period for plasticity (Hensch, 2005). In addition to vision, PNNs may also close the critical periods of plasticity in other sensory systems (McRae et al., 2007).

In contrast to sensory systems, little is known regarding the regulation of critical periods in sensorimotor systems and the behaviors that they underlie. Are PNNs involved in critical periods for vocal learning, such as those for birdsong and human speech? Humans and songbirds learn their vocalizations early in life during a two-part sensitive period consisting of an early perceptual phase during which species-typical sounds are memorized, followed by a later sensorimotor imitative phase during which auditory feedback is used to shape emitted sounds (Konishi, 1965; Doupe and Kuhl, 1999). Because of the many similarities between human speech and birdsong (Marler, 1970; Doupe and Kuhl, 1999), the oscine songbird provides a good model for investigating the neuronal mechanisms that underlie behavioral sensitive periods. The song nucleus HVC (this acronym is the proper name) is the nexus of auditory and motor pathways of the song system. Recent data from HVC suggest cellular parallels between the sensorimotor period of the song system and the visual critical period: HVC premotor activity is developmentally regulated (Crandall et al., 2007a), and these changes in activity relate to behavior (Day et al., 2008, 2009). Similar to the visual cortex during ocular dominance plasticity, the immature song nucleus HVC exhibits prolonged bursting. In addition, fastspiking putative interneurons fire before, during, and after song behavior during sensorimotor learning but not later in development (Crandall et al., 2007a). In the current study, we examined song system expression and regulation of two previously identified critical period indicators, PV and PNNs. 


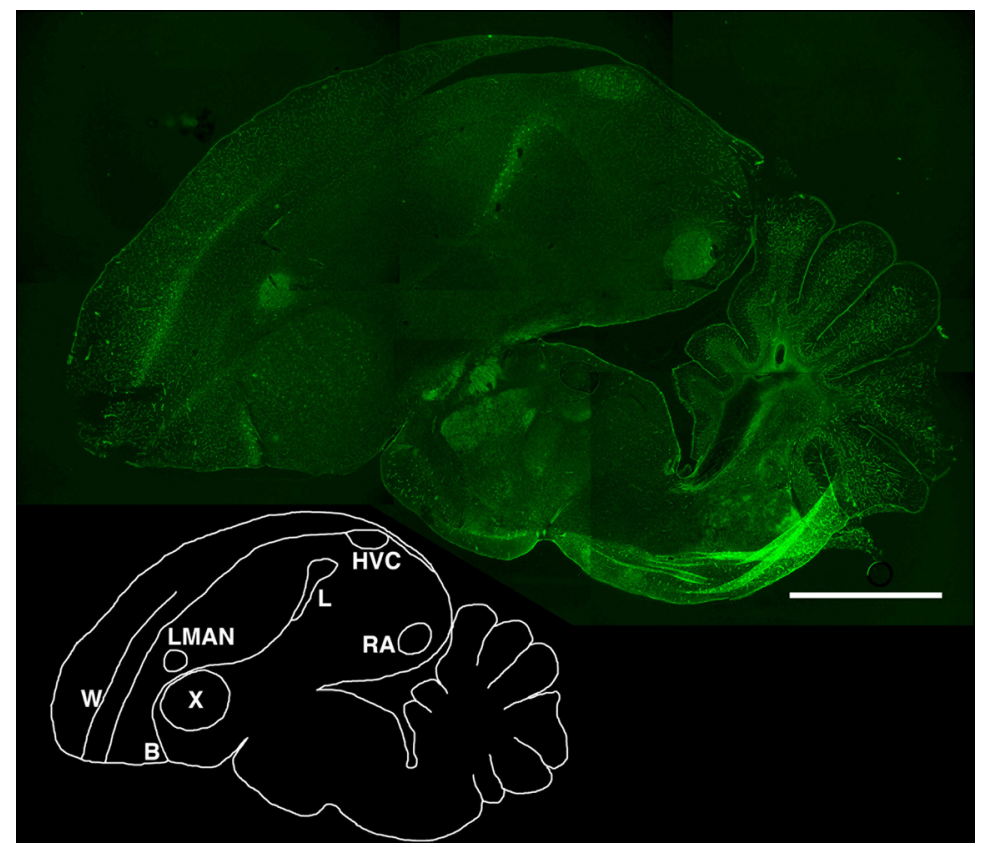

Figure 1. Perineuronal nets are found in the songbird brain and notably demarcate song nuclei and primary sensory areas. A parasagittal section of an adult finch brain was stained with an antibody to chondroitin sulfate, which labels PNNs. In the epifluorescence photomontage, PNNs are labeled in the brainstem, thalamus, primary pallial sensory areas, and song nuclei. PNN stain demarcates the boundaries of the song nuclei HVC, RA, LMAN, and Area X. PNN stain also highlights the primary sensory pallial areas field $\mathrm{L}$ (L; auditory), wulst (W; visual), and nucleus basalis (B; somatosensory). Anterior is left, and dorsal is up. Scale bar, $2 \mathrm{~mm}$.

were collected with a Carl Zeiss Axioskop and Jenoptik ProgRes MFcool camera. In some cases, brightness and contrast for a given set of panels were adjusted equally for all images stained with the same label with Adobe Photoshop (Adobe Systems).

Sampling strategy. Sections were sampled using a random, systematic sampling scheme throughout the mediolateral extent of each hemisphere. For adult-juvenile comparisons, every fifth section was taken with a random start between sections 20 and 25 (inclusive) to 100 from the midline. For an additional higherresolution LMAN study, every second section was taken with a random start between sections 30 and 32 (inclusive) to 80 from the midline. For the isolate study, every third section was taken with a random start between sections 20 and 23 (inclusive) to 100 from the midline. The random systematic sampling ensured that every part of each nucleus had an equal probability of being sampled. Each brain slice was coded with a random number. Sections from all ages (experiment 1, $33 \mathrm{~d}$ old, $65 \mathrm{~d}$ old, or adult) or treatments (experiment 2, isolate or control) were mixed in groups of two to three individuals for histology and stereology and randomly ordered such that the person counting was blind to age/treatment and could not make inferences about one section based on another.

To be included in our analysis, at least five

\section{Materials and Methods}

Subjects and histology. The 46 zebra finches (Taeniopygia guttata) in this study were reared in our facility on a $13 / 11 \mathrm{~h}$ light/dark cycle. Eight juvenile (33 d after hatching) and seven adult (143-330 d after hatching) male zebra finches were used in the adult-juvenile comparisons, six $33 \mathrm{~d}$ olds and four adults (150-318 d after hatching) were used for the higher-resolution lateral magnocellular nucleus of the anterior nidopallium (LMAN) study, nine 65-d-old finches were used in the songperineuronal net study, and 12 90-d-old finches (five isolates, three sibling controls, and four other controls) were used for the isolate study. For histology, all animals were deeply anesthetized with an overdose of sodium pentobarbital and perfused through the heart with $0.9 \% \mathrm{NaCl}$, followed by $2 \%$ paraformaldehyde or, for the isolate study, $4 \%$ paraformaldehyde with $0.2 \%$ Triton X-100 (Sigma) in $0.1 \mathrm{~m}$ phosphate buffer, $\mathrm{pH}$ 7.4. Brains were removed from the skull and cryoprotected in $30 \%$ sucrose for $24-48 \mathrm{~h}$ and then snap frozen in a cryostat and sectioned at $35 \mu \mathrm{m}$ in the sagittal plane. Sampling was done in a systematic-random manner. Brains from each age/treatment group were processed in tan$\mathrm{dem}$, using the same solutions. Sections were incubated $1 \mathrm{~h}$ at room temperature in a mixture of a monoclonal mouse anti-chondroitin sulfate antibody (CS56, 1:500; Sigma-Aldrich) and a polyclonal rabbit anti-PV antibody (1:1000, ab11427; Abcam) in 0.05 m TBS with $0.4 \%$ Triton X-100. Immunoreactivity was revealed by a $30 \mathrm{~min}$ incubation in a mixture of secondary antibodies: goat anti-mouse Alexa fluor 488 (1:100; Invitrogen) and goat anti-rabbit Alexa fluor 555 (1:200; Invitrogen) in $0.05 \mathrm{M}$ TBS with $0.4 \%$ Triton $\mathrm{X}-100$. To ensure that all structures considered $\mathrm{PV}$ positive $\left(\mathrm{PV}^{+}\right)$and/or $\mathrm{PNN}^{+}$were neurons, sections were then incubated for $30 \mathrm{~min}$ in Neurotrace 435/465 Nissl stain (1:500; Invitrogen) in $0.05 \mathrm{M}$ TBS. Nissl stains distinct nucleoli in neurons but not glia. Both the omission of the primary antibodies and preincubation in chondroitinase $A B C(0.5 \mathrm{U} / \mathrm{ml}$; Sigma) in $50 \mathrm{~mm}$ Tris base, $60 \mathrm{~mm}$ sodium acetate, and $0.02 \% \mathrm{BSA}, \mathrm{pH}$ 8.0 , at $37^{\circ} \mathrm{C}$ for $90 \mathrm{~min}$ resulted in the absence of chondroitin sulfate labeling (supplemental Fig. 1, available at www.jneurosci.org as supplemental material).

Confocal images were collected in $z$-stacks with a scanning laser confocal microscope (Olympus Fluoview FV1000). Epifluorescence images sections from both hemispheres combined had to contain the nucleus of interest. For the every fifth section adult-juvenile comparison, these criteria resulted in the exclusion of four $33 \mathrm{~d}$ olds from the robust nucleus of the arcopallium (RA) analysis, three $33 \mathrm{~d}$ olds from the Area $\mathrm{X}$ analysis, all $33 \mathrm{~d}$ olds from the LMAN analysis, and one $33 \mathrm{~d}$ old from the HVC analysis. With these criteria, one $65 \mathrm{~d}$ old was excluded from the songHVC comparison described below. Because all $33 \mathrm{~d}$ olds were excluded from the original LMAN analysis, a second higher-resolution study of LMAN that sampled every other section was executed.

Counting method. The brain slices were placed on a Carl Zeiss Axioskop and imaged using a $2.5 \times$ EC Plan-NEOFLUAR objective and analyzed with a Jenoptik ProgRes MFcool camera. After placing the grid over a given song nucleus, a grid square over the song nucleus was randomly selected by a random number generator (Matlab; MathWorks). From this initial randomly selected square, every second square within the nucleus was also counted, except in the relatively large song nucleus Area $\mathrm{X}$, in which every third square was counted. Using a $40 \times$ PlanNEOFLUAR objective on the Carl Zeiss Axioskop (described above) and a $75 \times 75 \mu \mathrm{m}$ unbiased counting frame, neuron counts were made using nucleoli as the unit of count throughout the entire depth of the section, as described previously (Tramontin et al., 1998). Identification of neurons based on Nissl-labeled nucleolar staining was immediately followed by the identification of PNNs and PV-positive cells in the same section. For counting purposes, a PNN was defined as a CS56-labeled relatively continuous oval ( $\geq 75 \%$ stain defining an oval shape) surrounding a previously marked nucleolus in the same focal plane. Parvalbumin-positive cells were identified by PV fluorescent stain surrounding a previously marked nucleolus in the same plane.

Brain area volume analysis. For volume analysis, a sampling grid was randomly oriented on the monitor, and each nucleus was outlined on the grid. The area of each square on the grid was $75 \times 75 \mu \mathrm{m}\left(5625 \mu \mathrm{m}^{2}\right)$. The number of gridline intersections that fell within the nucleus defined by Nissl staining were counted and analyzed following the Cavalieri principle (Gundersen and Jensen, 1987).

Song behavior study. Songs of nine $65 \mathrm{~d}$ olds were recorded for at least $3 \mathrm{~d}$ before the animals were killed. Sound data were sorted according to temporal properties. Preliminary songs were defined as sounds lasting 
$\geq 500 \mathrm{~ms}$ with time gaps of no more than 20 $\mathrm{ms}$. The songs were then segmented into syllables, and syllable features were calculated using Sound Analysis Pro (Tchernichovski et al., 2000). The median of each feature was compared with the percentage of $\mathrm{PV}^{+} \mathrm{HVC}$ neurons with PNNs.

Isolate study. Isolates were reared without a song tutor in an acoustic isolation chamber. The father was removed at day 10 and the mother and all siblings at day 27. Sibling controls were reared without the father from days 10 to 27 . Finches were killed on day 90 . Histology and stereology proceeded as described above. Fluorescence intensity was measured blind to condition and normalized to a brain area below HVC (the HVC shelf) that is stained weakly and indistinguishably from surrounding pallium. Both measurement areas (HVC and shelf) were $4032 \mu \mathrm{m}^{2}$.

Statistical analysis and data presentation. All data were analyzed with custom-written Matlab functions and Sound Analysis Pro (Tchernichovski et al., 2000). Data are presented as mean \pm SD. Histological data were compared with an unpaired Student's $t$ test (two-tailed). Behavioral and histological data were analyzed with a linear regression. Significance was defined as $p<0.05$.

\section{Results}

Critical period markers are found in the song system

Prompted by the physiological similarities between the visual and vocal sensitive periods (Crandall et al., 2007a), we studied cellular anatomical indicators of critical periods in the song system. We examined parasagittal brain slices from adults and juveniles near the onset of singing (aged $33 \mathrm{~d}$ ). We triple labeled each section with Nissl stain (which distinctly labels nucleoli of neurons but not glia) and antibodies to parvalbumin $(\alpha-\mathrm{PV})$ and chondroitin sulfate $(\alpha-$ $\mathrm{CS})$, a component of perineuronal nets.

In adult finches, primary sensory areas and song nuclei were brightly stained with $\alpha$-CS relative to surrounding areas (Fig. 1). The $\alpha$-CS label was specific (supplemental Fig. 1, available at www.jneurosci.org as supplemental material), because the stain was absent when the slice was labeled only with secondary antibody or preincubated with chondroitinase, an enzyme that degrades chondroitin-containing proteoglycans (Galtrey et al., 2008). For stereological analysis, a PNN was defined as a CSpositive annulus (at least 75\% complete) that surrounded a Nisslpositive distinct nucleolus. Higher-resolution images of $\alpha$-CS stain in song nuclei show the distinct PNNs, which were found around PV-expressing neurons in all song nuclei (Fig. 2) (supplemental Figs. 2-4, available at www.jneurosci.org as supplemental material). Three primary thalamorecipient areas contained PNNs (Fig. 1): the visual wulst, the somatosensory nucleus basalis, and the auditory field L2A (supplemental Fig. 5, available at www.jneurosci.org as supplemental material). PNNs were noted in the pallial (cortical) song nuclei HVC (Figs. 1, 2), RA, LMAN, and nucleus interfacialis (supplemental Fig. 2, available at www.jneurosci.org as supplemental material). The basal ganglia song nucleus Area X (Figs. 1, 2) contained dispersed but brightly stained PNNs. The two thalamic song nu-
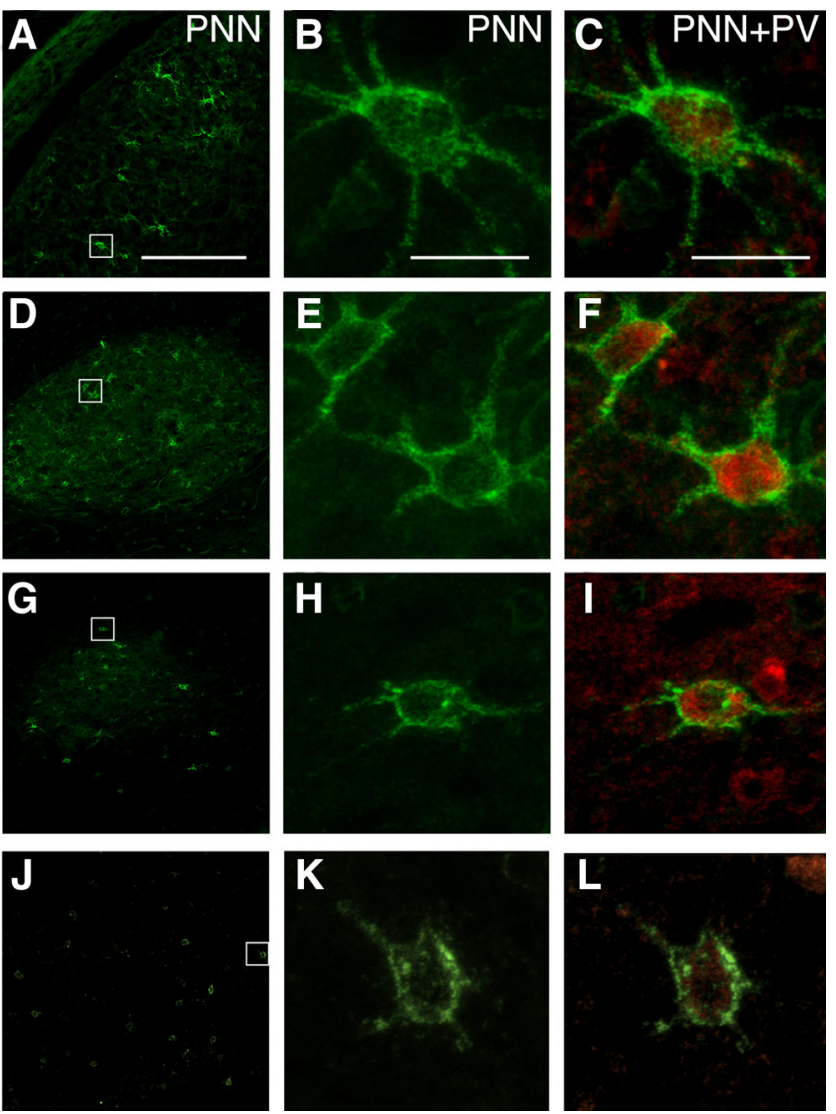

Figure 2. As in mammals, PNNs in the adult song system surround neurons that contain the calcium-binding protein parvalbumin. $A-L$, Each row contains representative data from a single song nucleus at low (column 1; scale bar, $250 \mu \mathrm{m}$ ) and higher Columns 1 and 2 show PNNs as revealed by $\alpha$-CS label, whereas column 3 shows the overlay of PNN and parvalbumin stains. Note the double-labeled cells in all song nuclei in column 3.

clei, nucleus uvaeformis (supplemental Fig. 3, available at www.jneurosci.org as supplemental material) and the medial dorsolateral nucleus of the anterior thalamus (supplemental Fig. 4, available at www.jneurosci.org as supplemental material), also contained PNNs.

To quantify the percentages of PNNs relative to total neurons and $\mathrm{PV}^{+}$neurons, we used an unbiased counting method that enabled systematic sampling of sections that were triple stained with Nissl and the two antibodies ( $\alpha$-CS and $\alpha$-PV). Four song nuclei are amenable to stereological analysis as a result of their morphological characteristics: HVC, RA, Area X, and LMAN. Our quantification yielded adult song nuclei volumes similar to previous reports (Table 1) (MacDougall-Shackleton et al., 1998; Gil et al., 2006), which indicated that our random samples accurately sampled entire nuclei. Because the relatively small nucleus LMAN was sampled at every other section whereas the other song nuclei were sampled at every fifth section, the data from LMAN cannot be directly compared with data from the other nuclei. Only a small percentage of total Nisslidentified adult neurons in song nuclei were surrounded by PNNs (Table 1) (supplemental Fig. 6, available at www.jneurosci.org as supplemental material). At least half of all PNNs surrounded PV ${ }^{+}$ neurons in all four nuclei (Table 1). More than $80 \%$ of the PNNs in HVC surrounded $\mathrm{PV}^{+}$neurons, and HVC contained the highest percentage of $\mathrm{PV}^{+}$neurons with PNNs. Collectively, these data indicate that PNNs are expressed in the adult song system, that their expression pattern varies across song nuclei, and that they preferentially surround neurons that contain parvalbumin. 
Table 1. Critical period markers in juvenile and adult song nuclei

\begin{tabular}{|c|c|c|c|c|c|c|c|}
\hline Song nucleus & $33 \mathrm{~d}$ olds and adults & Volume $\left(\mathrm{mm}^{3}\right)$ ( $n$ birds) & $\% \mathrm{PV}^{+}$and $\mathrm{PNN}^{+}$neurons & $\% \mathrm{PV}^{+}$neurons & $\% \mathrm{PNN}^{+}$neurons & \%PV with PNN & \%PNN with PV \\
\hline \multirow[t]{2}{*}{ HVC } & 33 & $0.053 \pm 0.016^{* *}(7)$ & $0.4 \pm 0.2^{* *}(6)$ & $9.1 \pm 5.3(6)$ & $0.6 \pm 0.7^{* *}$ & $4.8 \pm 6.3^{* *}(6)$ & $27.8 \pm 44.3^{*}(6)$ \\
\hline & Adult & $0.188 \pm 0.024^{* *}(7)$ & $3.3 \pm 0.3^{* *}$ & $8.1 \pm 2.4$ & $3.8 \pm 0.1^{* *}$ & $42.5 \pm 11.7^{* *}$ & $86.4 \pm 6.8^{*}$ \\
\hline \multirow[t]{2}{*}{ RA } & 33 & $0.058 \pm 0.014^{*}(5)$ & $1.3 \pm 0.9^{*}(4)$ & $19.4 \pm 10.3(4)$ & $1.5 \pm 1.8^{*}$ & $11.6 \pm 13.4(4)$ & $47.7 \pm 55.2(4)$ \\
\hline & Adult & $0.158 \pm 0.027^{*}(7)$ & $4.2 \pm 0.7^{*}$ & $18.9 \pm 7.3$ & $6.4 \pm 3.2^{*}$ & $23.0 \pm 6.4$ & $67.7 \pm 10.6$ \\
\hline \multirow[t]{2}{*}{ Area $X$} & 33 & $0.291 \pm 0.057^{*}(5)$ & $0.7 \pm 0.4(4)$ & $5.0 \pm 0.8^{* *}(4)$ & $1.1 \pm 0.9$ & $13.7 \pm 14.1^{*}(4)$ & $58.2 \pm 27.4(4)$ \\
\hline & Adult & $0.876 \pm 0.116^{*}(6)$ & $0.9 \pm 0.1$ & $2.9 \pm 0.4^{* *}$ & $1.8 \pm 0.5$ & $31.4 \pm 9.4^{*}$ & $50.7 \pm 10.3$ \\
\hline \multirow[t]{2}{*}{ LMAN } & 33 & $0.0575 \pm 0.022(6)$ & $1.2 \pm 0.3^{*}$ & $3.4 \pm 1.5$ & $1.8 \pm 0.7$ & $35.1 \pm 22.6$ & $63.3 \pm 25.0$ \\
\hline & Adult & $0.0476 \pm 0.007(4)$ & $2.4 \pm 0.4^{*}$ & $5.1 \pm 2.4$ & $2.8 \pm 0.8$ & $48.1 \pm 11.3$ & $83.2 \pm 11.5$ \\
\hline
\end{tabular}

Data indicate mean \pm SD. If not otherwise noted, the number of animals available for percentage calculations was the same as for volume. For given nucleus and measurement, adults and $33 \mathrm{~d}$ olds were significantly different $\left({ }^{*} p<0.05\right.$ ${ }^{* *} p<0.0005$, unpaired two-tailed $t$ test).
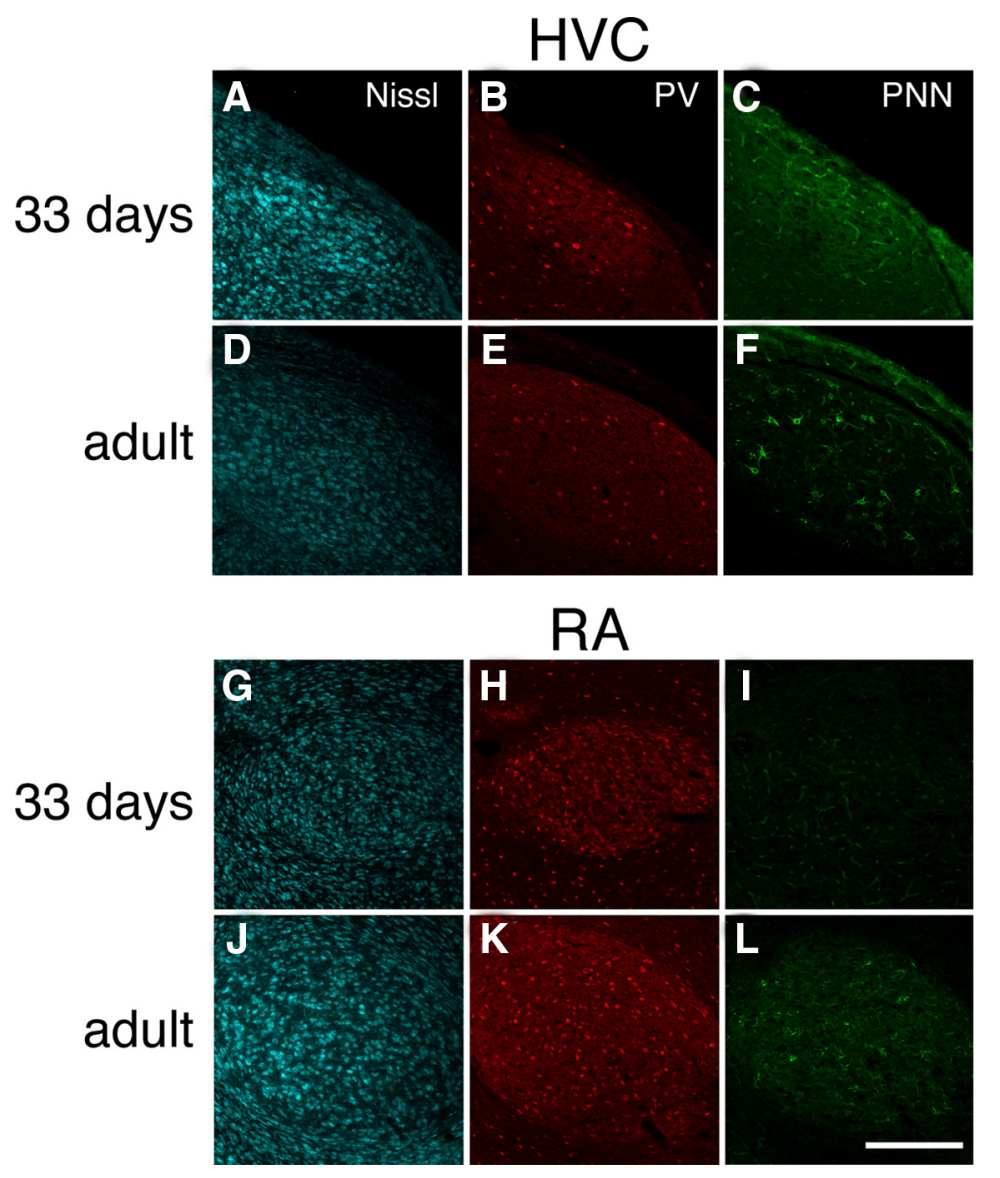

Figure 3. Song system perineuronal nets are developmentally regulated. Data are shown from two of the four song nuclei examined, but the same trends in PNN expression were seen in all (Table 1) (supplemental Figs. 7, 8, available at www.jneurosci. org as supplemental material). Representative confocal data from the HVCs of a $33-d-0$-old juvenile $(\boldsymbol{A}-\boldsymbol{C})$ and an adult $(\boldsymbol{D}-\boldsymbol{F})$ show the same section stained and imaged for $\operatorname{Niss}(\boldsymbol{A}, \boldsymbol{D}), \alpha-\operatorname{PV}(\boldsymbol{B}, \boldsymbol{E})$, and $\alpha-C S(\boldsymbol{C}, \boldsymbol{F})$. Note the developmental increase in $\operatorname{PNNs}(\boldsymbol{C}, \boldsymbol{F})$. RA PNNs were also developmentally regulated $(\mathbf{G}-\boldsymbol{L})$. Representative confocal data from the RAs of a $33-$ d-old juvenile $(\mathbf{G}-\boldsymbol{I})$ and adult $(\boldsymbol{J}-\boldsymbol{L})$ show the same section stained and imaged for Nissl $(\boldsymbol{G}, \boldsymbol{J}), \alpha$-PV $(\boldsymbol{H}, \boldsymbol{K})$, and $\alpha$-CS $(\boldsymbol{I}, \boldsymbol{L})$. Note the developmental increase in PNNs $(\boldsymbol{I}, \boldsymbol{L})$. Scale bar, $250 \mu \mathrm{m}$.

\section{Critical period markers are regulated during vocal learning}

If PNNs close the critical period for vocal learning, then PNN levels should be higher in adults than in juveniles that exhibit vocal plasticity. To test this hypothesis, we compared song system PNN expression in $33 \mathrm{~d}$ olds near the onset of singing with adults that expressed crystallized song. For HVC, RA, Area X, and LMAN, we quantified total volumes and percentages of PNNs around total and $\mathrm{PV}^{+}$neurons. As with previous results (Bottjer et al., 1985; Hermann and Bischof, 1986), we found that the volumes of HVC, RA, and Area X increased with development (Table 1; Fig. 3) (supplemental Fig. 7, available at www.jneurosci.org as supplemental mate- rial). Consistent with a previous finding (Bottjer et al., 1985), LMAN volume slightly decreased with development (Table 1) (supplemental Fig. 8, available at www. jneurosci.org as supplemental material).

We found that percentage of total and $\mathrm{PV}^{+}$neurons with PNNs trended to increase in all four of the song nuclei that were stereologically examined (Table 1). In $\mathrm{HVC}$, the percentage of total and $\mathrm{PV}^{+}$ neurons with PNNs significantly increased with development (Table 1; Fig. 3). Similarly, in RA, Area X, and LMAN, the percentage of total neurons with PNNs and the percentage of $\mathrm{PV}^{+}$neurons with PNNs trended to increase, and these differences were significant in several cases (Table 1; Fig. 3) (supplemental Figs. 7,8 , available at www.jneurosci.org as supplemental material). Interestingly, the percentage of total Area X neurons that was positive for PV significantly decreased with development (Table 1) (supplemental Fig. 7, available at www.jneurosci.org as supplemental material). We noted that the $\alpha$-CS "background" staining trended to be higher in juveniles than adults (Fig. 3, $C$ vs F). This apparent change may have functional implications, because CS proteoglycans have opposite effects on neuron process outgrowth depending on whether they are assembled as barriers such as PNNs or as a uniform substrate (Dityatev et al., 2007). Collectively, these data indicate that the PNN expression increases during vocal sensorimotor learning in telencephalic song nuclei and that there are differences in PNN and PV expression among nuclei.

Critical period markers correlate with song maturity

The observed differences in PV and PNN expression between adults and juveniles could be related to vocal learning or, alternatively, to a developmental process that occurs regardless of the status of learned song. To investigate possible relationships between song and these critical period markers, we recorded song and stained the brains from $65 \mathrm{~d}$ olds that were approximately halfway through the sensorimotor phase (Fig. 4). We focused our analysis on HVC because its size and obvious borders in 


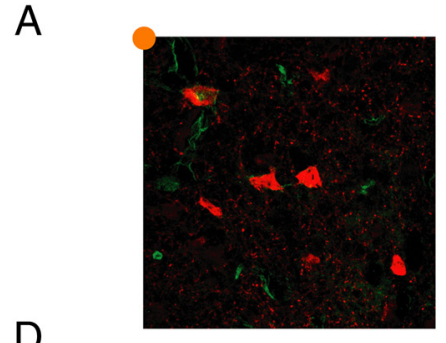

$\mathrm{D}$

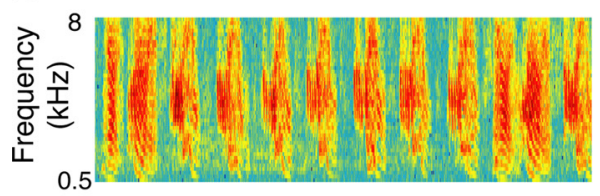

F Entropy Variance
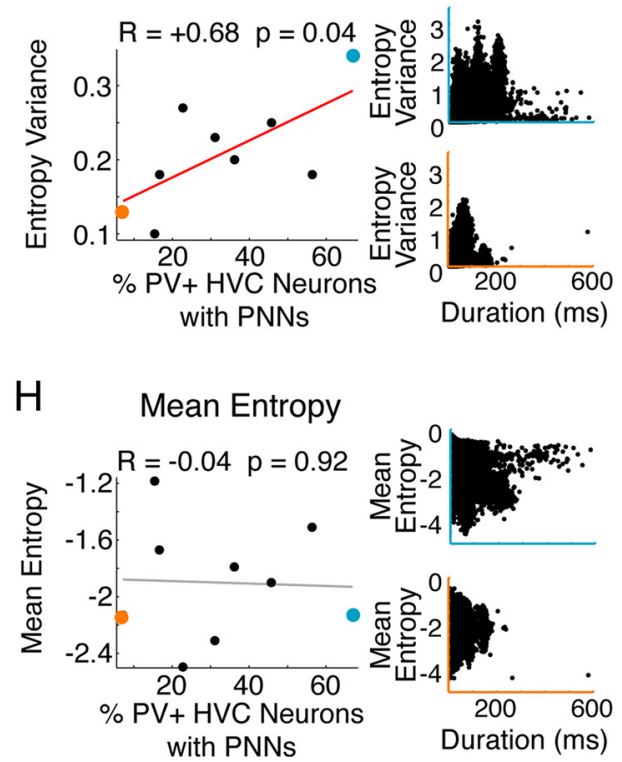
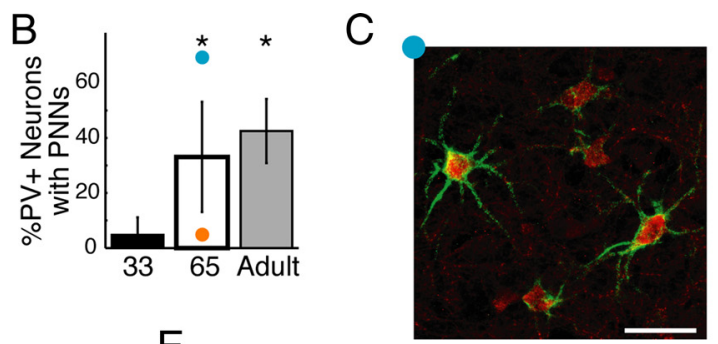

$\mathrm{E}$

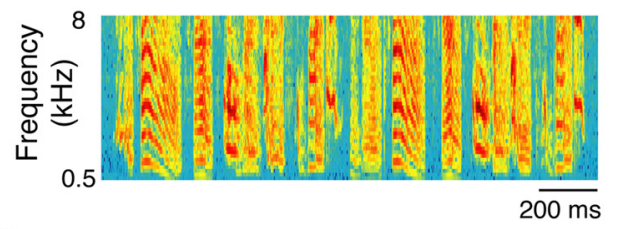

G

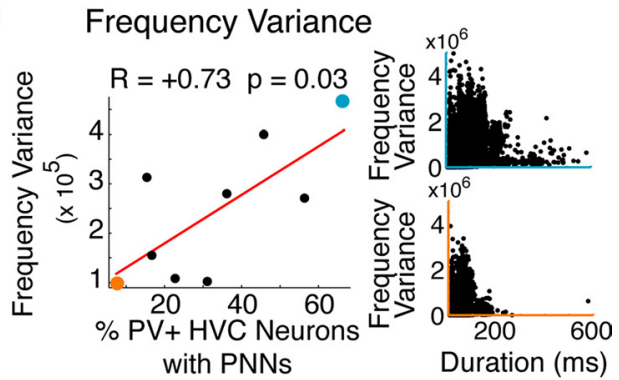

I

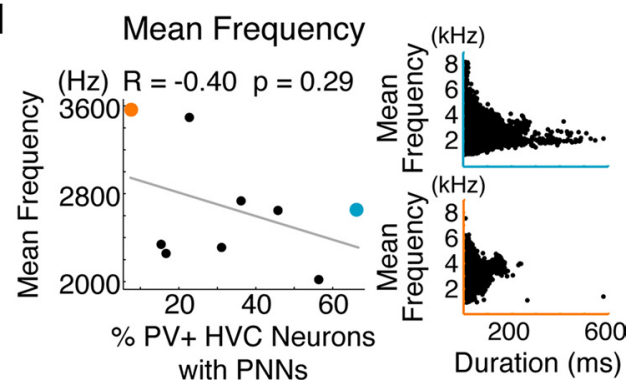

Figure 4. HVC perineuronal nets predict the maturity of song, as measured by temporal variance. $A, C$, The percentage of parvalbumin-positive HVC neurons (red) with PNNs (green) varied in 65-d-old zebra finches. Representative micrographs are shown for a juvenile HVC that had either a low $(\boldsymbol{A} ; 7.2 \%)$ or high $(\boldsymbol{C} ; 66.7 \%)$ percentage of PNNs around PV ${ }^{+}$neurons. The orange and cyan dots in the top left corners correspond to the colors in $\boldsymbol{B}$ and $\boldsymbol{F}-I$. Scale bar, $50 \mu \mathrm{m} . \boldsymbol{B}, \operatorname{In} 65 \mathrm{~d}$ olds, the percentage of PV ${ }^{+}$neurons with PNNs was highly variable and significantly different from $33 \mathrm{~d}$ olds but not adults. The data from the finches in $\boldsymbol{A}$ and $\boldsymbol{C}$ are shown in orange and cyan, respectively. $\boldsymbol{D}, \boldsymbol{E}$, Representative sonograms of the same juveniles with the corresponding histology data directly above suggest that fewer PNNs correlate with simpler songs. Note the simple, repetitive syllable in $\boldsymbol{D}$ produced by the finch with few PNNs in $\boldsymbol{A}$. Correspondingly, note the more complex song in $\boldsymbol{E}$ from the finch with more PNNs in $\boldsymbol{C . F}, \mathbf{G}$, Each panel quantifies the relationship of the percentage of PV ${ }^{+} \mathrm{HV}$ ( neurons with PNNs to different spectral features of song syllables (left, medians for each finch for each syllable feature). The colored data correspond to the specific finches shown in $\boldsymbol{A}-\boldsymbol{E}$. On the right, the feature data for each syllable produced by these specific finches are shown relative to duration. The following song features are shown: entropy variance $(\boldsymbol{F})$, mean frequency variance $(\boldsymbol{G})$, mean entropy $(\boldsymbol{H})$, and mean frequency $(\boldsymbol{I})$. Note that the temporal variance of both entropy $(\boldsymbol{F})$ and mean frequency $(\boldsymbol{G})$ were significantly correlated with PNNs.

Nissl-stained sections reliably enabled inclusion in stereological analyses, it exhibited the largest relative changes in PNNs with development, and, within 33-d-old and adult groups, measures of PNN percentages were in general less variable than other song nuclei. In $65 \mathrm{~d}$ olds halfway through the sensorimotor sensitive period, however, the percentage of $\mathrm{PV}^{+} \mathrm{HVC}$ neurons with PNNs was highly variable and significantly different from 33-dold finches but not adults (Fig. $4 B$ ).

In the $65 \mathrm{~d}$ olds, we directly compared the PNN pattern to song maturity, finch by finch. Increasing temporal variance of spectral features was used previously to indicate the maturation of song (Tchernichovski et al., 2000; Derégnaucourt et al., 2005). We therefore compared PV and PNN expression in HVC with the temporal variance of song, as measured by the variance of Weiner entropy and mean frequency across syllables. We found that the percentage of $\mathrm{PV}^{+} \mathrm{HVC}$ neurons with PNNs predicted the tem- poral variance of song. Figure $4, A$ and $C$, shows overlaid micrographs of $\alpha$-PV (red) and $\alpha$-CS (green) stain of HVCs from two $65 \mathrm{~d}$ olds. Note the differences in PNN expression, with very little CS stain in Figure 4A, but large PNNs in Figure 4C. Example songs from these two animals recorded before they were killed are shown below each respective micrograph in Figure 4, $D$ and $E$. A simple song (Fig. $4 D$ ) was produced by the finch with few PNNs (Fig. $4 \mathrm{~A}$, indicated by orange in figures below), whereas a more complex song (Fig. $4 E$ ) was produced by the finch with larger and more numerous PNNs (Fig. 4C, indicated by cyan in figures below). This effect was quantified across animals by plotting the percentage of $\mathrm{PV}^{+} \mathrm{HVC}$ neurons with PNNs against two measures of temporal complexity: the entropy variance and the mean frequency variance. The medians of these features across all syllables are plotted against the percentage of $\mathrm{PV}^{+} \mathrm{HVC}$ neurons with PNNs. This measure of PNN expression significantly pre- 

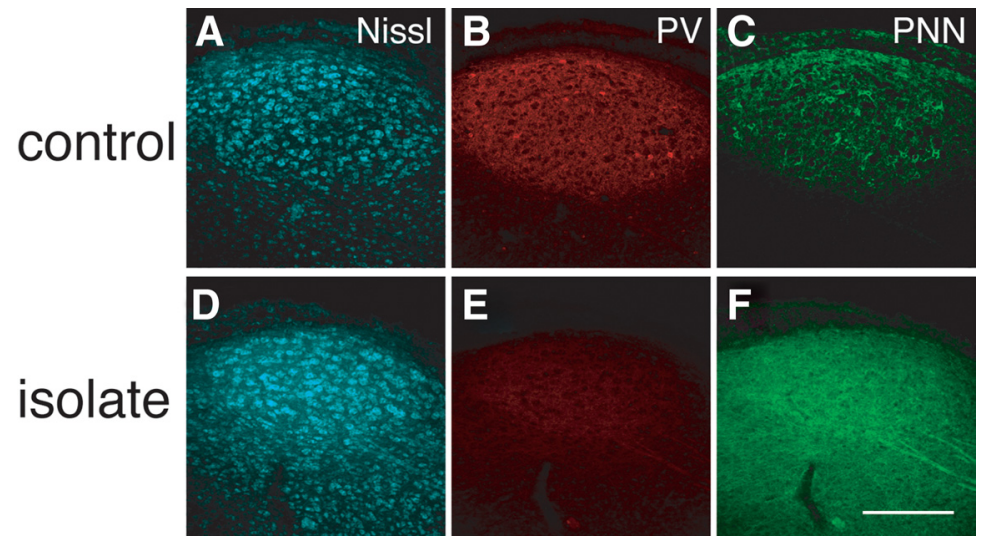

Figure 5. Isolation from a song tutor alters critical period indicators in HVC. Representative data from an age-matched control $(\boldsymbol{A}-\boldsymbol{C})$ and 90 -d-old isolate $(\boldsymbol{D}-\boldsymbol{F})$ are shown. The same section was triple stained and imaged for the following: Nissl (blue stain in $\boldsymbol{A}, \boldsymbol{D}), \mathrm{PV}$ (red stain in $\boldsymbol{B}, \boldsymbol{E}$ ), and PNNs (green stain in $\boldsymbol{C}, \boldsymbol{F}$ ). Note the lack of punctate PV-positive neurons in $\boldsymbol{E}$ and the less striking PNNs in $\boldsymbol{F}$. Scale bar, $250 \mu \mathrm{m}$.

Table 2. Critical period markers in the HVC of isolates and controls

\begin{tabular}{|c|c|c|}
\hline & Control & Isolate \\
\hline Volume $\left(\mathrm{mm}^{3}\right)(n)$ & $0.16 \pm 0.03^{*}(7)$ & $0.10 \pm 0.05^{*}(5)$ \\
\hline$\% \mathrm{PV}^{+}$and $\mathrm{PNN}^{+}$neurons & $0.76 \pm 0.41^{* *}$ & $0.20 \pm 0.20^{* *}$ \\
\hline$\% \mathrm{PV}^{+}$neurons & $0.94 \pm 0.44^{* *}$ & $0.29 \pm 0.30^{* *}$ \\
\hline$\% \mathrm{PNN}^{+}$neurons & $0.85 \pm 0.48$ & $0.71 \pm 0.36$ \\
\hline$\%$ PV with PNN & $79.58 \pm 15.66$ & $45.91 \pm 44.89$ \\
\hline \%PNN with PV & $90.29 \pm 9.41^{* *}$ & $28 \pm 28.44^{* *}$ \\
\hline$\%$ PNN without PV & $9.71 \pm 9.41^{* *}$ & $72.00 \pm 28.44^{* *}$ \\
\hline \multicolumn{3}{|l|}{ Intensity measurements } \\
\hline$\%$ Nissl intensity relative to background $(n)$ & $178.23 \pm 49.08(12)$ & $191.70 \pm 42.39(10)$ \\
\hline \%PV intensity relative to background & $220.18 \pm 41.58^{* *}$ & $165.64 \pm 35.92^{* *}$ \\
\hline$\% C S$ intensity relative to background & $291.00 \pm 84.88^{* *}$ & $203.30 \pm 48.45^{* *}$ \\
\hline
\end{tabular}

All animals were $90 \mathrm{~d}$ of age. Data indicate mean \pm SD. The number of animals available for percentage calculations was the same as for volume. Intensity $n$ values are noted in the Nissl intensity columns. For given nucleus and measurement, isolate and control were significantly different $\left({ }^{*} p<0.02\right.$, ${ }^{* *} p<0.004$, unpaired two-tailed $t$ test).

dicted temporal complexity of song (Fig. $4 F, G$ ). However, note that the $p$ values are 0.04 and 0.03 , respectively, which would not be significant with a Bonferroni's correction for multiple comparisons $(\alpha=0.0125)$. The percentage of $\mathrm{PV}^{+}$ neurons with PNNs did not predict other features of song syllables: mean entropy and mean frequency (Fig. $4 H, I$ ). These data reveal a correlation between $\mathrm{PNN}$ coating of $\mathrm{PV}^{+}$neurons and song maturation.

\section{Delaying the vocal sensitive period alters critical period markers}

Sensory deprivation decreases PV (Cellerino et al., 1992; Philpot et al., 1997) and PNNs (Sur et al., 1988) in affected sensory neural pathways. Does sensory deprivation also affect PV and PNNs in the song system, which is sensorimotor? To address this question, we deprived developing zebra finches of a song tutor through acoustic isolation, which delays the closure of the critical period for vocal learning (Eales, 1985). At 90 d of age, isolationperturbed finches and age-matched controls were killed, and their brains were triple stained for Nissl, PV, and CS as described above. In pilot studies, we noted that the intensity of isolate PNN pallial stain appeared more uniform than controls, with song areas not well defined (pilot data not shown, but see Fig. 5). To optimize tissue staining for quantification of intensity (see below), we lowered nonspecific background staining by including Triton X-100 (Sigma-Aldrich) in the fixation perfusion media
(Torres et al., 2006). Because this method increases the number of neurons counted (Torres et al., 2006), the percentage of total neurons that were $\mathrm{PV}^{+}$and $\mathrm{PNN}^{+}$ correspondingly decreased (compare $\% \mathrm{PV}^{+}$and $\% \mathrm{PNN}^{+}$neurons in adults and $90 \mathrm{~d}$ controls in Tables 1 and 2, respectively). As in previous studies (Leitner and Catchpole, 2007), isolation decreased the volume of the song nucleus HVC (Table 2). Stereological analysis revealed a nonsignificant trend of isolation to decrease the percentage of $\mathrm{PV}^{+}$neurons with PNNs (Table 2). More strikingly, the percentage of total HVC neurons that were $\mathrm{PV}^{+}$was significantly decreased by isolation (Table 2). Interestingly, the percentage of total neurons with PNNs did not change significantly with isolation (Table 2) $(p=0.58)$. Consistent with a decrease in the percentage of $\mathrm{PV}^{+}$neurons and no change in the percentage of neurons with PNNs, the percentage of PNNs surrounding PV-negative neurons increased with isolation (Table 2).

Although the relative number of neurons with PNNs did not significantly change, the relative intensity of PNNs, as well as PV, in $\mathrm{HVC}$ was affected by isolation (Fig. $5 F$ ). To quantify this effect, we measured intensity of PV and CS stain in the HVCs of isolates and controls. Intensity in HVC was measured relative to a control region in the same slice. Normalized intensity of PV and CS label were both significantly decreased in isolates relative to controls (Table 2). Collectively, these data indicate that isolation from a song tutor alters critical period indicators.

\section{Discussion}

The mechanisms that regulate sensory critical periods appear to involve the calcium-binding protein parvalbumin and extracellular matrix perineuronal nets (Pizzorusso et al., 2002; Hensch, 2005). We now report that PNNs appear in the right places (all seven song nuclei) at the right time (during song learning) to play a role in vocal learning in the zebra finch. We also report that, during song learning, the percentage of $\mathrm{PV}^{+}$neurons with PNNs in the song nucleus HVC predicts song maturity (as measured by temporal complexity). Furthermore, isolation from a song tutor decreases the number of $\mathrm{PV}^{+}$neurons and the relative intensity of both PV and a component of PNNs in HVC. Collectively, these data indicate cellular anatomical mechanisms that may regulate the sensitive period for vocal plasticity.

We have identified several characteristics of the plastic song system: (1) the song nucleus HVC expresses prolonged bursts before and after singing (Crandall et al., 2007a); (2) the prolonged bursting neurons are fast-spiking putative interneurons, and these cells are the only HVC neurons that are activated by sensory stimulation (auditory playback) in the awake juvenile (Crandall et al., 2007a); (3) HVC bursting during sleep parallels song development and predicts decreased overnight song plasticity (Crandall et al., 2007b); (4) PV expression is decreased by tutor song deprivation (current study); and (5) PNNs appear during the sensorimotor phase, correlate with song maturity, and are affected by tutor song deprivation (present study). Our data suggest that similar, although less striking, developmental changes occur in song nuclei other than HVC. This is consistent with previous studies (Tramontin et al., 2003) and suggests that the regulation of song plasticity is distributed. Comparison of the 
birdsong sensorimotor phase and the visual critical period prompted the current study. Likewise, the similarities between the vocal sensitive period and the critical period for ocular dominance plasticity may inform studies of visual plasticity. For example, direct comparison of the two systems would suggest that bursting in the visual cortex during sleep may increase during the critical period for ocular dominance plasticity and underlie the differential plasticity effects of sleep across developmental stages (Frank et al., 2001).

As with human speech (Marler, 1970; Doupe and Kuhl, 1999), birdsong is acquired in two developmental stages: a sensory phase during which species-specific sounds are memorized and a sensorimotor phase during which vocalizations are actively sculpted toward the learned model using auditory feedback (Konishi, 1965; Bolhuis and Gahr, 2006). In the zebra finch, these two stages overlap. We found that, at the onset of the sensorimotor phase in normally reared animals (33 d), parvalbumin expression is already at or above adult levels. In contrast, tutor song deprivation with acoustic isolation significantly decreased the number of $\mathrm{PV}^{+}$neurons. These data suggest that typical PV expression in HVC requires sensory input from a song tutor, possibly combined with social interactions (Boseret et al., 2006). Surprisingly, the number of total HVC neurons with PNNs was not affected by tutor deprivation, although the relative intensity was significantly decreased. This suggests that a population of HVC neurons eventually develop rudimentary PNNs regardless of tutor song experience. Thus, PNNs, which are thought to close critical periods (Hensch, 2005), appear in the absence of the typical developmental increase in PV, which is thought to open critical periods. These data are consistent with cellular and behavioral data that indicate that the sensitive period for vocal learning can be delayed but will eventually close even when developmental milestones are not achieved (Doupe and Kuhl, 1999; Yazaki-Sugiyama and Mooney, 2004).

PNNs appear to stabilize sensory circuits and affect neuronal excitability (Hockfield and McKay, 1983; Zaremba et al., 1989; Dityatev et al., 2007). PNNs increase at the end of sensory critical periods and depend on activity (Hensch, 2005). If PNNs are similar in their regulation and function in sensory and sensorimotor systems, then the PNNs may stabilize the neural circuits involved in song learning. The PNNs are found throughout the song system, which then suggests that the entire song system is stabilized during or after developmental song learning. PNNs affect the electrical excitability of the neurons they surround, most prominently PV-positive fast-spiking neurons (Härtig et al., 1999), which are typically interneurons (but RA-projecting HVC neurons also express PV) (Wild et al., 2005). If PNNs affect song system excitability, then song system excitability should change during the period in which they appear (between $33 \mathrm{~d}$ and adulthood). In support of this hypothesis, previous work in the song system has shown that fast-spiking HVC neurons alter their activity during song learning (Crandall et al., 2007a). Developmental cellular changes in HVC are reflected in premotor activity bursts that decrease in duration (Crandall et al., 2007a) and become stronger (Day et al., 2009) during the sensorimotor phase. These stronger bursts appear to stabilize behavior (Day et al., 2008, 2009).

If PNNs stabilize the song system and, correspondingly, song behavior, then destruction of the PNNs with chondroitinase should reopen the period of plasticity, as achieved previously in the visual system (Pizzorusso et al., 2002). However, extensive preliminary investigations have not revealed a simple and direct relationship between song system PNNs and song plasticity (our unpublished observations). This may be attributable to the failure to reactivate additional critical period mechanisms, such as changes in excitatory-inhibitory balance, as described previously for the visual system (Morishita and Hensch, 2008) and/or additional complicating factors in a sensorimotor versus sensory system. Future experiments may require a combined manipulation of PNNs and neural activity to induce changes in behavior. If the vocal learning critical period for songbirds can be reopened with a combination of chondroitinase and perturbation of neural activity, the possibility exists that the vocal learning critical period could also be opened in humans. The ability to reopen the critical period for speech would provide a new avenue for treatments of developmental communication disorders.

Atypical development of interneurons is implicated in several neurodevelopmental disorders, including autism (Di Cristo, 2007), suggesting that understanding interneurons and their roles in plasticity will illuminate human disease. Interneurons and PNNs appear to have roles in the regulation of sensory critical periods (Hensch, 2005) and vocal learning in the songbird (Crandall et al., 2007a; present study) (Y. Yazaki-Sugiyama, J. Kushner, N. A. Hessler, and T. K. Hensch, personal communication). The songbird model provides a unique opportunity to investigate the effects of interneuron regulation on the development of a quantifiable behavior. Previous work has already shown that HVC premotor population bursts, which are dominated by interneuron activity (Rauske et al., 2003; Crandall et al., 2007a), are developmentally regulated (Crandall et al., 2007a; Day et al., 2009) and appear to orchestrate the degree of vocal plasticity online, millisecond by millisecond (Day et al., 2008). It is possible that, similar to mammalian cortex (Driver and Frith, 2000), the regulation of sensory access to HVC is regulated by oscillatory bursting activity that is controlled by interneurons (Buzsáki and Chrobak, 1995; Tamás et al., 2000; Galarreta and Hestrin, 2001). As a consequence, HVC premotor bursting may prevent sensoryguided plasticity (Engel et al., 2001). If so, weak top-down influences (in the form of prolonged bursts with low firing rates) may be critical for sensorimotor learning (Engel et al., 2001; Buschman and Miller, 2007). During the sensorimotor period, we speculate that PNNs progressively stabilize HVC premotor bursting. If this hypothesis is applicable to humans, it suggests that a subset of communication disorders may result from a premature stabilization of oscillatory bursting activity in vocal control areas or, in other diseases, failure to stabilize bursting activity during vocal practice. Consistent with a premature increase in the strength and stability of oscillations and corresponding decrease in plasticity (Day et al., 2008, 2009), autistics express relatively rigid, stereotyped behaviors, impairment in perceptual selection (Belmonte et al., 2004), and atypical brain oscillatory activity (Cantor et al., 1986; Eigsti and Shapiro, 2003; Stroganova et al., 2007). The songbird system presents an excellent model in which to further examine the mechanisms of critical period regulation in a behavioral model.

\section{References}

Belmonte MK, Cook EH Jr, Anderson GM, Rubenstein JL, Greenough WT, Beckel-Mitchener A, Courchesne E, Boulanger LM, Powell SB, Levitt PR, Perry EK, Jiang YH, DeLorey TM, Tierney E (2004) Autism as a disorder of neural information processing: directions for research and targets for therapy. Mol Psychiatry 9:646-663.

Bolhuis JJ, Gahr M (2006) Neural mechanisms of birdsong memory. Nat Rev Neurosci 7:347-357.

Boseret G, Carere C, Ball GF, Balthazart J (2006) Social context affects testosterone-induced singing and the volume of song control nuclei in male canaries (Serinus canaria). J Neurobiol 66:1044-1060. 
Bottjer SW, Glaessner SL, Arnold AP (1985) Ontogeny of brain nuclei controlling song learning and behavior in zebra finches. J Neurosci 5:1556-1562.

Braun K, Scheich H, Zuschratter W, Heizmann CW, Matute C, Streit P (1988) Postnatal development of parvalbumin-, calbindin- and adult GABA-immunoreactivity in two visual nuclei of zebra finches. Brain Res 475:205-217.

Buschman TJ, Miller EK (2007) Top-down versus bottom-up control of attention in the prefrontal and posterior parietal cortices. Science 315:1860-1862.

Buzsáki G, Chrobak JJ (1995) Temporal structure in spatially organized neuronal ensembles: a role for interneuronal networks. Curr Opin Neurobiol 5:504-510.

Cantor DS, Thatcher RW, Hrybyk M, Kaye H (1986) Computerized EEG analyses of autistic children. J Autism Dev Disord 16:169-187.

Celio MR (1986) Parvalbumin in most gamma-aminobutyric acidcontaining neurons of the rat cerebral cortex. Science 231:995-997.

Cellerino A, Siciliano R, Domenici L, Maffei L (1992) Parvalbumin immunoreactivity: a reliable marker for the effects of monocular deprivation in the rat visual cortex. Neuroscience 51:749-753.

Crandall SR, Aoki N, Nick TA (2007a) Developmental modulation of the temporal relationship between brain and behavior. J Neurophysiol 97:806-816.

Crandall SR, Adam M, Kinnischtzke AK, Nick TA (2007b) HVC neural sleep activity increases with development and parallels nightly changes in song behavior. J Neurophysiol 98:232-240.

Day NF, Kinnischtzke AK, Adam M, Nick TA (2008) Top-down regulation of plasticity in the birdsong system: "premotor" activity in the nucleus HVC predicts song variability better than it predicts song features. J Neurophysiol 100:2956-2965.

Day NF, Kinnischtzke AK, Adam M, Nick TA (2009) Daily and developmental modulation of "premotor" activity in the birdsong system. Dev Neurobiol 69:796-810.

Derégnaucourt S, Mitra PP, Fehér O, Pytte C, Tchernichovski O (2005) How sleep affects the developmental learning of bird song. Nature 433:710-716.

Di Cristo G (2007) Development of cortical GABAergic circuits and its implications for neurodevelopmental disorders. Clin Genet 72:1-8.

Dityatev A, Brückner G, Dityateva G, Grosche J, Kleene R, Schachner M (2007) Activity-dependent formation and functions of chondroitin sulfate-rich extracellular matrix of perineuronal nets. Dev Neurobiol 67:570-588.

Doupe AJ, Kuhl PK (1999) Birdsong and human speech: Common themes and mechanisms. Annu Rev Neurosci 22:567-631.

Driver J, Frith C (2000) Shifting baselines in attention research. Nat Rev Neurosci 1:147-148.

Eales LA (1985) Song learning in zebra finches: some effects of song model availability on what is learnt and when. Anim Behav 33:1293-1300.

Eigsti IM, Shapiro T (2003) A systems neuroscience approach to autism: biological, cognitive, and clinical perspectives. Ment Retard Dev Disabil Res Rev 9:205-215.

Engel AK, Fries P, Singer W (2001) Dynamic predictions: Oscillations and synchrony in top-down processing. Nat Rev Neurosci 2:704-716.

Fagiolini M, Hensch TK (2000) Inhibitory threshold for critical-period activation in primary visual cortex. Nature 404:183-186.

Frank MG, Issa NP, Stryker MP (2001) Sleep enhances plasticity in the developing visual cortex. Neuron 30:275-287.

Galarreta M, Hestrin S (2001) Spike transmission and synchrony detection in networks of GABAergic interneurons. Science 292:2295-2299.

Galtrey CM, Kwok JC, Carulli D, Rhodes KE, Fawcett JW (2008) Distribution and synthesis of extracellular matrix proteoglycans, hyaluronan, link proteins and tenascin- $\mathrm{R}$ in the rat spinal cord. Eur $\mathrm{J}$ Neurosci 27: 1373-1390.

Gil D, Naguib M, Riebel K, Rutstein A, Gahr M (2006) Early condition, song learning, and the volume of song brain nuclei in the zebra finch (Taeniopygia guttata). J Neurobiol 66:1602-1612.

Gundersen HJ, Jensen EB (1987) The efficiency of systematic sampling in stereology and its prediction. J Microsc 147:229-263.

Härtig W, Derouiche A, Welt K, Brauer K, Grosche J, Mäder M, Reichenbach A, Brückner G (1999) Cortical neurons immunoreactive for the potas- sium channel Kv3.1b subunit are predominantly surrounded by perineuronal nets presumed as a buffering system for cations. Brain Res 842:15-29.

Hensch TK (2005) Critical period plasticity in local cortical circuits. Nat Rev Neurosci 6:877-888.

Herrmann K, Bischof HJ (1986) Delayed development of song control nuclei in the zebra finch is related to behavioral development. J Comp Neurol 245:167-175.

Hockfield S, McKay RD (1983) A surface antigen expressed by a subset of neurons in the vertebrate central nervous system. Proc Natl Acad Sci U S A 80:5758-5761.

Knudsen EI (2004) Sensitive periods in the development of the brain and behavior. J Cogn Neurosci 16:1412-1425.

Konishi M (1965) The role of auditory feedback in the control of vocalization in the white-crowned sparrow. Z Tierpsychol 22:770-783.

Leitner S, Catchpole CK (2007) Song and brain development in canaries raised under different conditions of acoustic and social isolation over two years. Dev Neurobiol 67:1478-1487.

MacDougall-Shackleton SA, Hulse SH, Ball GF (1998) Neural correlates of singing behavior in male zebra finches (Taeniopygia guttata). J Neurobiol 36:421-430.

Marler P (1970) Birdsong and speech development: could there be parallels? Am Sci 58:669-673.

McRae PA, Rocco MM, Kelly G, Brumberg JC, Matthews RT (2007) Sensory deprivation alters aggrecan and perineuronal net expression in the mouse barrel cortex. J Neurosci 27:5405-5413.

Morishita H, Hensch TK (2008) Critical period revisited: impact on vision. Curr Opin Neurobiol 18:101-107.

Philpot BD, Lim JH, Brunjes PC (1997) Activity-dependent regulation of calcium-binding proteins in the developing rat olfactory bulb. J Comp Neurol 387:12-26.

Pizzorusso T, Medini P, Berardi N, Chierzi S, Fawcett JW, Maffei L (2002) Reactivation of ocular dominance plasticity in the adult visual cortex. Science 298:1248-1251.

Rauske PL, Shea SD, Margoliash D (2003) State and neuronal class-dependent reconfiguration in the avian song system. J Neurophysiol 89:1688-1701.

Stroganova TA, Nygren G, Tsetlin MM, Posikera IN, Gillberg C, Elam M, Orekhova EV (2007) Abnormal EEG lateralization in boys with autism. Clin Neurophysiol 118:1842-1854.

Sur M, Frost DO, Hockfield S (1988) Expression of a surface-associated antigen on Y-cells in the cat lateral geniculate nucleus is regulated by visual experience. J Neurosci 8:874-882.

Tamás G, Buhl EH, Lörincz A, Somogyi P (2000) Proximally targeted GABAergic synapses and gap junctions synchronize cortical interneurons. Nat Neurosci 3:366-371.

Tchernichovski O, Nottebohm F, Ho CE, Pesaran B, Mitra PP (2000) A procedure for an automated measurement of song similarity. Anim Behav 59:1167-1176.

Torres EM, Meldrum A, Kirik D, Dunnett SB (2006) An investigation of the problem of two-layered immunohistochemical staining in paraformaldehyde fixed sections. J Neurosci Methods 158:64-74.

Tramontin AD, Smith GT, Breuner CW, Brenowitz EA (1998) Seasonal plasticity and sexual dimorphism in the avian song control system: stereological measurement of neuron density and number. J Comp Neurol 396:186-192.

Tramontin AD, Wingfield JC, Brenowitz EA (2003) Androgens and estrogens induce seasonal-like growth of song nuclei in the adult songbird brain. J Neurobiol 57:130-140.

Wiesel TN, Hubel DH (1963) Single-cell responses in striate cortex of kittens deprived of vision in one eye. J Neurophysiol 26:1003-1017.

Wild JM, Williams MN, Howie GJ, Mooney R (2005) Calcium-binding proteins define interneurons in HVC of the zebra finch (Taeniopygia guttata). J Comp Neurol 483:76-90.

Yazaki-Sugiyama Y, Mooney R (2004) Sequential learning from multiple tutors and serial retuning of auditory neurons in a brain area important to birdsong learning. J Neurophysiol 92:2771-2788.

Zaremba S, Guimaraes A, Kalb RG, Hockfield S (1989) Characterization of an activity-dependent, neuronal surface proteoglycan identified with monoclonal antibody Cat-301. Neuron 2:1207-1219. 